\title{
Precursors of an upcoming solar cycle at high latitudes from coronal green line data
}

\author{
K. Petrovay, M. Nagy, T. Gerják \\ EötvösUniversity, Department of Astronomy, Budapest, Hungary \\ L. Juhász \\ EötvösUniversity, Department of Geophysics and Space Science, Budapest, Hungary
}

\begin{abstract}
After reviewing potential early indicators of an upcoming solar cycle at high latitudes, we focus attention on the rush-to-the-poles (RTTP) phenomenon in coronal green line emission. Considering various correlations between properties of the RTTP with the upcoming solar cycle we find a correlation between the rate of the RTTP and the time delay until the maximum of the next solar cycle. On the basis of this correlation and the known internal regularities of the sunspot number series we predict that, following a minimum in 2019, cycle 25 will peak in late 2024 at an amplitude of about 130 (in terms of smoothed monthly revised sunspot numbers). This slightly exceeds the amplitude of cycle 24 but it would still make cycle 25 a fairly weak cycle.

Keywords: Sun, solar activity, solar cycle, sunspot number
\end{abstract}

\section{Introduction}

Solar Cycle 25 reached its maximum in April 2014 with a 13-month smoothed sunspot number of $R=116$ (revised value $\oint^{1}$ ) or $R=70$ (unrevised value), about

\footnotetext{
${ }^{*}$ Corresponding author

Email address: K.Petrovay@astro.elte.hu (K. Petrovay)

${ }^{1}$ The revision of the official sunspot number series that took place in 2015 was a well known crucial milestone in recent solar physics. For more explanations see http://sidc.oma.be/silso/newdataset
}

Preprint submitted to Journal of ${ }^{A} T_{E} X$ Templates

February 16, 2018 
$30 \%$ lower than an average cycle and roughly half the typical amplitude of cycles 18-23, comprising the Modern Maximum. As common with solar cycles, the current cycle was double peaked: the main maximum was preceded by a lower secondary maximum in March 2012. The two maxima were associated with a phase shift between the hemispheres, the first and second maxima corresponding to peak activity in the Northern and Southern hemisphere, in September 2011 and April 2014, respectively.

The evident end of the Modern Maximum has triggered increased interest in the underlying causes of intercycle variations in solar activity and in forecasting methods (Petrovay 2010). However, despite extensive efforts, attempts to forecast an upcoming solar cycle significantly earlier than its start have not been successful. The most successful methods of solar cycle prediction are either based on the Waldmeier effect (relation between the rise rate of activity in the early phase of a solar cycle to its maximum), for which the cycle must have already started, or on the polar precursor method (a link between indicators of the magnetic field at the poles of the Sun, peaking around solar minimum, and the amplitude of the next maximum).

Both of these methods suggest that the most promising place on the Sun to look for even earlier precursors of an upcoming cycle is at high heliographic latitudes. On the one hand, solar cycles are known to overlap by up to $\sim$ 1-2 years on the sunspot butterfly diagram and possibly up to $\sim 5-6$ years on the butterfly diagrams of other solar features such as torsional oscillations, ephemeral active regions or coronal line emission. On the other hand, the polar magnetic field is observed to be built up from the poleward transport of following $(f)$ polarity magnetic flux from decaying active regions by meridional flows and turbulent diffusion, traced by a high-latitude poleward branch in the butterfly diagram of various solar features such as polar faculae and coronal line emission. Detailed studies of the evolution of the latitudinal distribution of these proxies may therefore potentially yield some early signs of the new solar cycle in the making.

In the present paper, after briefly reviewing information about some other 
potential proxies, we will focus on coronal green line emission data, in particular to the poleward branch on the butterfly diagram of this emission, also known as the "rush to the poles" (RTTP). As the green line emission in this branch is thought to trace the closed magnetic field structures just outside the boundary of the polar coronal hole, the RTTP is a manifestation of the decrease of poloidal magnetic flux due to the poleward spread of opposite polarity flux from the $f$ polarity parts of active regions of the ongoing cycle. Indeed, soon after the RTTP finally reaches the pole the polar magnetic field is reversed, and, as a consequence of further flux transport from the lower latitudes, starts to increase in amplitude again until it reaches its maximum around the following sunspot minimum. This raises the question whether properties of the RTTP can give us a hint regarding the following increase of the polar field to its maximum, and, by implication, regarding the timing and amplitude of the next sunspot maximum.

Section 2 reviews information about other high-latitude magnetic proxies in view of forecasting possibilities for cycle 25 . Section 3 presents the green line data used, our methods of processing these data, and the results, while Section 4 concludes the paper.

\section{The high-latitude magnetic field and its proxies}

In this section we review information about other high-latitude magnetic proxies in view of forecasting possibilities for cycle 25. For further and less cycle-specific information we refer the reader to the reviews of Petrie (2015) and Petrie et al. (2014).

\subsection{Polar magnetic fields}

Direct measurements of the magnetic field strength near the Sun's poles have been made on a daily basis at Wilcox Solar Observatory since 1976. What is measured is the mean value of the line-of-sight field component in the polemost aperture of the instrument, covering roughly the area polewards of $55^{\circ}$ latitude. 


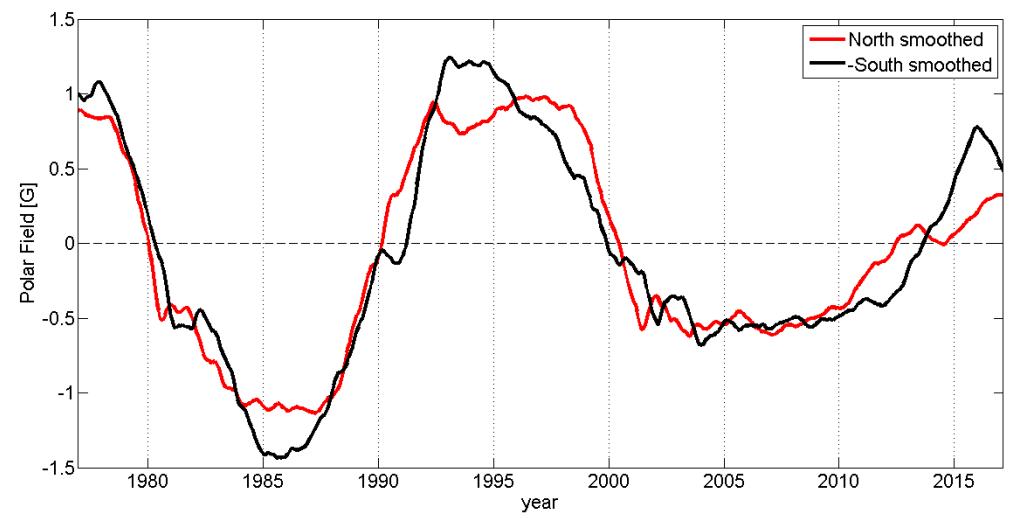

Figure 1: WSO polar magnetic field strength smoothed with a 13-month sliding window for the $\mathrm{N}$ (red) and S (black) hemispheres.

Upon passing data through a low-pass window to filter out annual variations due to the tilt of the Sun's axis and averaging between hemispheres, the resulting polar field amplitude is found to have peaked in the range $0.65-1.31 \mathrm{G}$ for the last four solar minima. The WSO polar field strength last reversed in March 2013 (one year after the peak of sunspot acitvity in the $\mathrm{N}$ hemisphere and one year ahead of the peak in the $\mathrm{S}$ hemisphere). Considering the two hemispheres separately, the reversal in the Northern hemishphere took place in May 2012 while in the South it occurred in July 2013 (Fig. 1 and Table 1). In the 4 years elapsed since the reversal, the polar field reached a peak value of $0.54 \mathrm{G}$ in February 2016, significantly below even the lowest previous peak of $0.65 \mathrm{G}$ in the last solar minimum. While, based on previous experience, the current decrease of the polar field may still be reversed and a higher maximum value may be reached, in none of the three previous solar minima did the peak WSO polar field strength exceed the highest value measured in the first 4 years after reversal by more than $\sim 20 \%$. This suggests that the peak polar field during the upcoming solar minimum may not exceed the value of 0.65 measured in the last minimum. As the peak polar magnetic field has been shown to correlate with the amplitude of the following sunspot maximum, by inference we may conclude 
that cycle 25 is unlikely to be significantly stronger than cycle 24 . Instead it may peak at amplitude similar to or slightly lower than the current cycle.

It should be noted that the reversal of the polar field measured at WSO is not, strictly speaking, the actual polar reversal. Even in the case of an axially symmetric magnetic field distribution, the mean field poleward of $55^{\circ}$ latitude reverses when the magnetic neutral line is still at a considerable distance from the pole. An alternative method to define the moment of field reversal is to consider the moment when the axial dipole moment (as determined from a spherical harmonic decomposition) of the global solar magnetic field distribution changes sign. On the basis of their analysis of SDO HMI magnetograms, Sun et al. (2015) report that this took place in October 2013; the mean field above $60^{\circ}$ latitude reversed in November 2012 and March 2014 on the N and S hemispheres, respectively.

$\mathrm{H}_{\alpha}$ filaments and filament channels serve as a good proxy tracing the position of the neutral line. Filament observations at Kislovodsk indicate that the neutral line finally reached the N pole in July 2013 and the S pole in December 2014.

Magnetic measurements at WSO and with the newly installed Routine Prediction Solar Telescope (RPST) at Kislovodsk have shown an interesting phenomenon: while the magnetic field strength in the South polar region has been increasing at a steady rate since the polar reversal, in the North polar region it has lingered at very low values in the first six months following reversal before starting to increase at a slower rate compared to the South. As a result, the magnetic field strength at $\mathrm{N}$ pole is still significantly weaker compared to the South, a situation somewhat analoguous to the minimum prior to cycle 23 . Nevertheless, in cycle 23 the activity level in the two hemispheres did not prove to be very different and it actually peaked two years earlier in the North.

\subsection{Torsional oscillations}

The realization that the equatorward migration of a pair of belts of alternating fast and slow rotation (known as torsional oscillation) starts at latitudes $\sim 50^{\circ}-60^{\circ}$ many years before the appearance of the first sunspots in the region 
Table 1: Milestones of solar cycle 24

\begin{tabular}{|c|c|c|c|c|}
\hline Latitude & North & South & Overall & \\
\hline Sunspot maximum & 2011.7 & 2014.3 & 2014.3 & SILSO WDC (2017) \\
\hline Reversal $>55^{\circ}$ lat. $(\mathrm{WSO})$ & 2012.5 & 2013.6 & \multirow{3}{*}{$\begin{array}{r}2013.3 \\
2013.8^{a}\end{array}$} & Scherrer et al. (1977) \\
\hline Reversal $>60^{\circ}$ lat. (HMI) & 2012.9 & 2014.3 & & Sun et al. $(2015)$ \\
\hline Reversal at $90^{\circ}$ lat. (Kislovodsk) & 2013.6 & 2014.9 & & Tlatov et al. (2015) \\
\hline
\end{tabular}

${ }^{a}$ global dipole

of maximal shear was what originally gave rise to the concept of an extended solar cycle in the 1980s (Wilson et al. (1988)). In this scenario the high-latitude component of the torsional oscillation pattern is interpreted as the extension of the low latitude pattern backward in time, implying that solar cycles are considerably longer than 11 years,with a long overlap between consecutive cycles. Indeed, the lower latitiude, fast-rotating member of the new pair appeared roughly 10 years before the start of cycle 24 . A few years ago considerable alarm was caused by the claim that no sign of the new belt pair that, in the extended solar cycle scenario, is taken to correspond to cycle 25 could be seen at high latitudes, despite being long overdue - some even envisioned the onset of a new grand minimum. It was, however, demonstrated by Howe et al. (2013) that if the reference background differential rotation profile used in the derivation of the rotational modulation is a mean calculated over a shorter time period, the torsional oscillation signal associated with cycle 25 is clearly seen starting from $\sim 2010$. At any rate, the detected signal is significantly weaker than it was in the case of cycle 24, possibly indicating a continued long term weakening of the solar activity level. Nevertheless, as helioseismic and surface Doppler data on the high latitude torsional oscillations (Ulrich et al. 1988, Howe et al. 2006) have only been available for a few solar cycles (cf. Fig. 30 in Howe 2009), far reaching conclusions regarding a relationship between this pattern and cycle amplitudes should be treated with caution. 


\subsection{Polar faculae}

Polar faculae are generally regarded to be a manifestation of the intermittent nature of the photospheric magnetic field at high latitudes, i.e. the equivalent of magnetic network elements at lower latitudes, with higher typical magnetic flux as a consequence of the higher $\sim 10 \mathrm{G}$ strength of the large scale field near the poles. This interpretation was recently empirically confirmed by Kaithakkal et al. (2013) on the basis of Hinode observations: nearly all magnetic patches with a flux above $10^{18} \mathrm{Mx}$ were found to be unipolar and to harbour polar faculae. A few faculae were found to correspond to minority polarity patches but these tend to have fluxes below $10^{18} \mathrm{Mx}$. (Blanco Rodríguez and Kneer 2010 report that $15 \%$ of polar faculae possess minority polarity magnetic field.)

All this implies that counts of polar faculae can be used as proxies of the polar magnetic field strength. Indeed, Muñoz-Jaramillo et al. (2012) report an excellent correlation between Mount Wilson Observatory polar facular counts (above latitudes of $70^{\circ}$ ) and WSO magnetic field strength measurements, effectively extending the polar magnetic field data set back to 1906. For a similar reconstruction based on Kodaikanal Ca II K data see Priyal et al. (2014).

\subsection{Ephemeral active regions}

Active regions tend to emerge at the latitude of maximal shear between the pair of fast and slow rotating belts of the torsional oscillation pair. This suggests that the pattern is either due to a magnetic quenching of the poleward angular momentum transport in the shallow layers of the convective zone (Petrovay and Forgács-Dajka 2002) or, indirectly, is a consequence of the Coriolis force acting on meridional inflows towards the activity belt caused by a magnetic suppression of the convective heat transport (Spruit 2003). Either way, one may expect that the backwards extension of the equatorward migrating torsional oscillation belt is also accompanied by the emergence of bipolar magnetic regions around the region of maximal shear. Before the appearance of the first sunspots of a cycle these regions can only be detected as ephemeral active regions (EARs). It was indeed found long ago by Martin and Harvey (1979) that high latitude ephemeral 
active regions tend to cluster on the backwards extension of the wings of the sunspot butterfly diagram (cf. also Hagenaar 2001).

More recently, in their analysis of Kitt Peak and MDI magnetograms for three solar cycles Tlatov et al. (2010) find that the first bipoles with orientation corresponding to an upcoming solar cycle appear at latitudes of $\sim 60^{\circ}$, shortly after the maximum of the previous cycle.

McIntosh et al. (2014) and McIntosh and Leamon (2017) study the migration pattern of EUV coronal bright points and of certain magnetogram features that they call g-nodes and consider to be tracers of bright points. In agreement with the extended cycle paradigm they find that the equatorward migration of these features starts at $55^{\circ}$ latitude many years before the appearance of the first sunspots of the subsequent cycle. By construction, their g-nodes are unipolar flux concentrations, so the relationship with EARs is not obvious.

By an ingenious method based on the proper motions of coronal jets, Savcheve, et al. (2009) also find that EARs in the polar coronal holes statistically follow Hale's polarity rule. Moreover, some time between early 2007 and late 2008 the orientation of these EARs changed from the one corresponding to cycle 24 to that of cycle 25. Early signs of the upcoming cycle are thus apparently observed at high latitudes already around the start of the previous cycle.

It should be noted that detailed analyses of Hinode vector magnetograms of the polar region (e.g. Shiota et al. (2012) ) do not show features that might be obviously identified with ephemeral active regions. As discussed above, magnetic patches with fluxes above $10^{18} \mathrm{Mx}$ are unipolar, while smaller flux elements do not show any significant dependence on cycle, contrary to what is expected for EAR. The solution of this puzzle is not known.

\section{Analysis of coronal green line emission data}

Emission from the solar corona is well known to be highly inhomogeneous, depending on magnetic topology and field strength. Measurements of the intensity of the coronal green line of Fe XIV at $5303 \AA$ slightly above the solar 
limb, as a function of position angle have been made on a regular basis for a period far exceeding the availability and life time of space observatories; as a consequence, long term variations in the corona covering several solar cycles are most commonly analyzed using green line emission data.

A homogeneous data base of green line emission intensities has been compiled by Minarovjech et al. (2011) and recently extended by cross calibration with SoHO EIT data (Dorotovič et al. 2014). Our investigation is based on this extended homogeneous data set, available in a public data base ${ }^{2}$

In this paper we focus on the RTTP due to its presumed link with the buildup of the poloidal field. For a quantitative study of the RTTP time-latitude diagrams ("butterfly diagrams") of the green line emission need to be constructed with sufficient contrast and detail at higher latitudes.

As high latitude structures in the corona are quite faint compared to the lowlatitude emission, simply plotting the detected intensity is not a suitable method for the study of the polar coronal structures. Alternative methods used by various authors for the visualization of these structures include plotting the count of local maxima in the positional angle distribution of the emission (e.g Tappin and Altrock 2013); plotting the standard deviation of the intensity relative to the mean value over a longer period (Leroy and Noens 1983); and applying an unsharp masking procedure to the butterfly diagram of the raw intensity (Robbrecht et al. 2010). Experimenting with all three methods we found that the RTTP manifests itself most clearly in plots made with the unsharp masking method, applying a masking window of width 25 degrees in latitude on the 11-month running mean of the raw intensity data, and then subtracting this masked (blurred) image from the original, to enhance fine details. The result is shown by contour lines in Fig. 2. In what follows, we will present results based on the data processed in this way.

In order to separate the polar component for quantitative analysis, the strongest positions above $60^{\circ}$ latitudes were identified. As starting point of

\footnotetext{
${ }^{2}$ http://www . suh.sk/online-data/modifikovany-homogenny-rad
} 


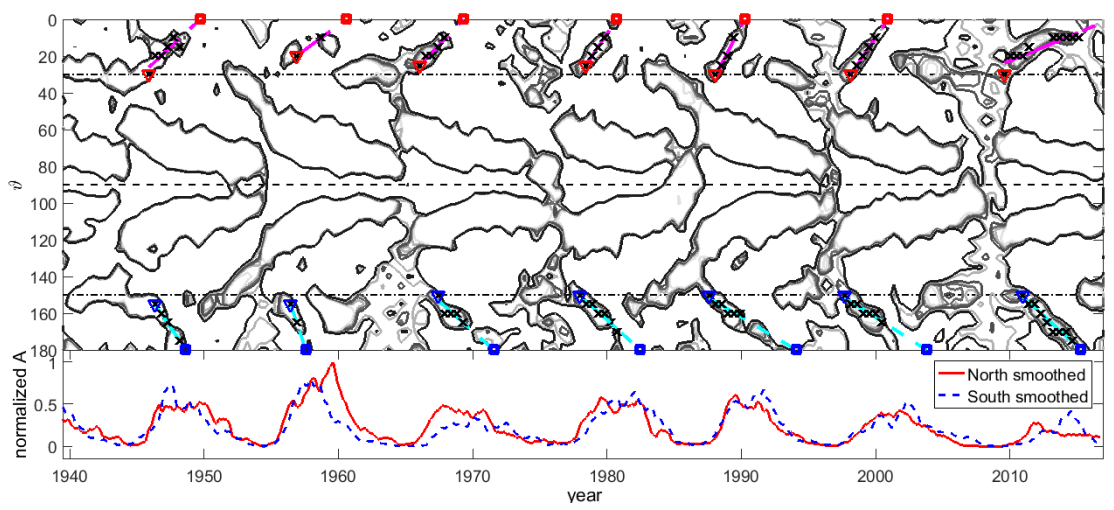

Figure 2: 11-month running averages of the coronal green line intensity after applying unsharp masking with a $25^{\circ}$ window in latitude (top panel). Crosses mark the position of the RTTP, triangle the beginning, square the end of RTTP. Dashed lines are linear fits to these points. The bottom panel shows the smoothed, normalized sunspot area for each hemisphere (http://solarscience.msfc.nasa.gov/greenwch.shtml).

RTTP component (crosses in Fig. 2) we chose the epoch after which the pattern starts to approach the pole (see triangles in Fig. 2). For each solar cycle and each hemisphere a linear fit was applied to these points to determine the end and tilt of the RTTP.

\section{Results}

The start and end dates of the RTTP as well as its tilt were plotted against a variety of solar cycle parameters including amplitude, rise rate or time to maximum. The best correlation $(r=0.66)$ is found in the case shown in Fig. 3

As some of the RTTP's marked out in Fig. 2 are rather uncertainly defined, we also attempted a weighted fit to the data points in Fig. 3, the weights being the number of latitude grid points for which RTTP positions (local maxima) were detected (crosses in Fig. 2). The resulting fit, however, was found to be almost indistinguishable to the one in Fig. 3 .

This correlation found between the rise rate of the RTTP and the time delay from the ending of RTTP to the maximum of the following cycle may be used 


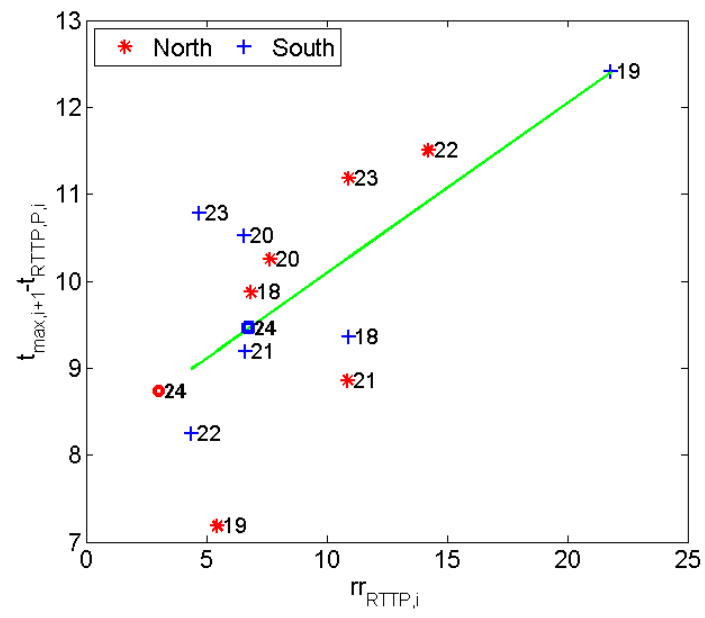

Figure 3: Rate of the RTTP $\operatorname{rr}_{\mathrm{RTTP}}$ (in degree/year) against time (in years) from the end of the RTTP $t_{\mathrm{RTTP}, i}$ to the next solar maximum $R_{\max , i+1}$ for individual cycles. The correlation coefficient is 0.66. The green line is a linear fit to the data. Circle and square mark the projections of the rate of the RTTP measured in cycle 24 ( $\mathrm{N}$ and S hemispheres) on this fit.

to attempt to predict the time of the maximum of cycle 25 . The fit shown in Fig. 3 may be written as

$$
t_{\text {max }, i+1}-t_{\mathrm{RTTP}, i}=a_{1} \operatorname{rr}_{\mathrm{RTTP}, i}+a_{2}
$$

where $t_{\max , i}$ and $t_{\mathrm{RTTP}, i}$ are the epochs of the maximum and of the end of the RTTP in cycle $i ; \operatorname{rr}_{\mathrm{RTTP}, i}$ is the rise rate of the RTTP in cycle $i$; while the $a_{i}$ 's are parameters of the fit.

Inserting into equation (1) the rate of the RTTP measured in cycle 24 in the Southern hemisphere (where it is better defined in this cycle) yields a time delay of 9.47 years from the time when the RTTP reached the poles to the next maximum. It follows that the maximum of cycle 25 is expected to occur in October 2024

\section{Conclusion}

Based on an analysis of the rush-to-the-poles phenomenon in coronal green line data we concluded that the rate of the RTTP is correlated with the time 

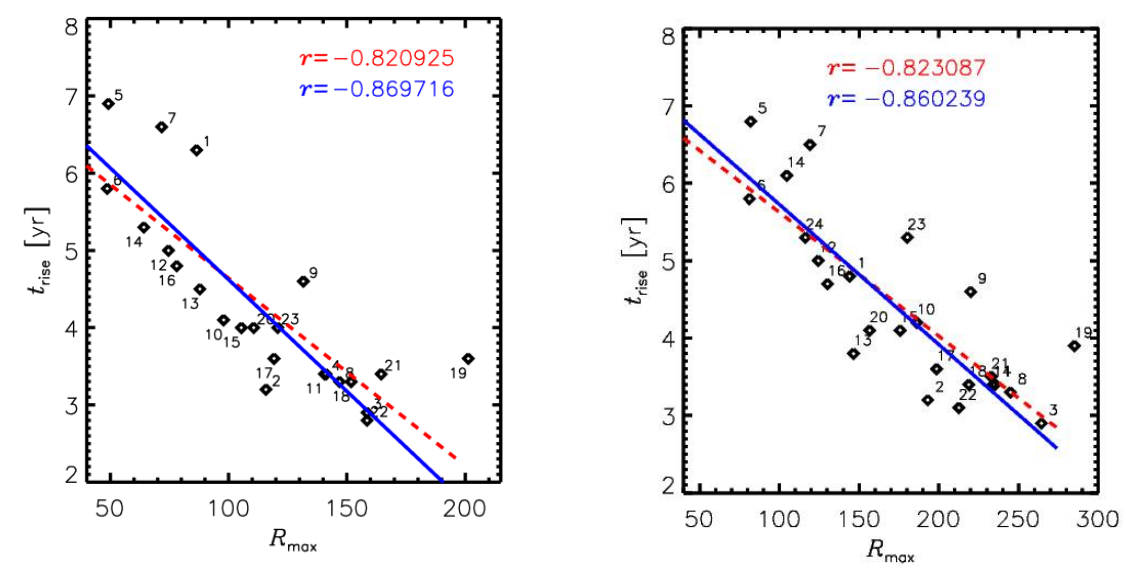

Figure 4: Correlation between cycle rise time $t_{\text {rise }}$ vs maximum amplitude $R_{\max }$ (Waldmeier effect) with unrevised (left) and revised (right) smoothed sunspot numbers. (Red dashed: fit to all data points; blue solid: cycle 19 treated as outlier.)
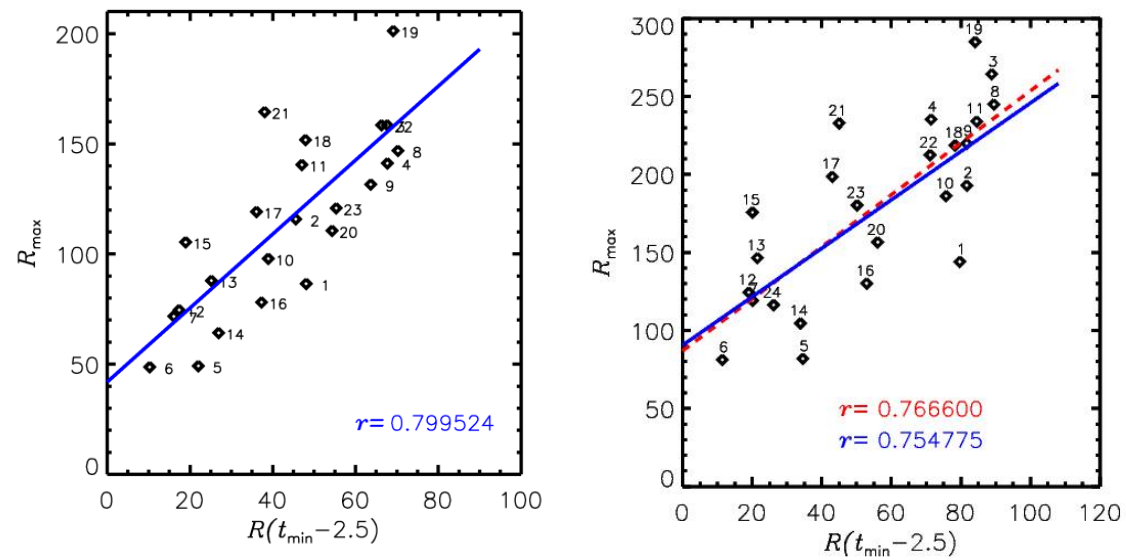

Figure 5: Correlation between maximum cycle amplitude $R_{\max }$ and sunspot number value 2.5 years before the previous minimum $R\left(t_{\min }-2.5\right)$ for unrevised (left) and revised (right) smoothed sunspot numbers. (Red dashed: fit to all data points; blue solid: cycle 19 treated as outlier.) 
delay to to the maximum of the next solar cycle counted from the time when the RTTP reaches the pole. On the basis of this result we predict the next solar maximum to to occur in October 2024.

In addition to the correlation found above, equation (1), two other, quite robust correlations exist between parameters of subsequent cycles (cf. Figs 4 and 5). One of these is the Waldmeier effect (Waldmeier 1935)

$$
t_{\max , i+1}-t_{\min , i+1}=a_{3} R_{\max , i+1}+a_{4}
$$

where $t_{\min , i}$ is the epoch of the minimum starting cycle $i$ while $R_{\max , i}$ is the maximum value of the smoothed sunpot number $R$ in the cycle. The other known correlation is the "minimax3" rule discussed in Petrovay (2010) and originally due to Cameron and Schüssler (2007):

$$
R_{\max , i+1}=a_{5} R\left(t_{\min , i+1}-2.5\right)+a_{6}
$$

where $R(t)$ is the smoothed sunspot number series, known from observations and here evaluated 2.5 years before the minimum.

Once we determined $t_{\max , i+1}$ from equation (1), a numerical solution of equations (2) and (3) yields $R_{\max , i+1}=129$ and $t_{\min , i+1}=2019.4$.

In summary, based on the apparent correlation between the rate ofthe RTTP and the time delay to the next maximum and the known internal regularities of the sunspot number series we predict that, following a minimum in 2019, cycle 25 will most likely peak in late 2024 at an amplitude of about 130 . This slightly exceeds the amplitude of cycle 24 but it would still make cycle 25 a fairly weak cycle.

\section{Acknowledgement}

This paper includes material first presented at the ISSI/VarSITI-SEE forum on "Expected evolution of solar activity in the following decades", held in March

2016 in Bern. The hospitality of the organizers is gratefully acknowledged. This research was partially funded by the European Union's Horizon 2020 research 
and innovation programme under grant agreement No. 739500. SILSO sunspot number data used in this study are provided by the Royal Observatory of Belgium, Brussels. Wilcox Solar Observatory data used in this study was obtained via the web site http://wso.stanford.edu/, courtesy of J.T. Hoeksema. The Wilcox Solar Observatory is currently supported by NASA.

\section{References}

Blanco Rodríguez, J., Kneer, F., 2010. Faculae at the poles of the Sun revisited: infrared observations. A\&A 509, A92. doi 10.1051/0004-6361/200811111.

Cameron, R., Schüssler, M., 2007. Solar cycle prediction using precursors and flux transport models. Astrophys. J. 659, 801-811. doi:10.1086/512049, arXiv:arXiv:astro-ph/0612693

Dorotovič, I., Minarovjech, M., Lorenc, M., Rybanský, M., 2014. Modified Homogeneous Data Set of Coronal Intensities. Solar Phys. 289, 2697-2703. doi:10.1007/s11207-014-0501-2.

Hagenaar, H.J., 2001. Ephemeral Regions on a Sequence of Full-Disk Michelson Doppler Imager Magnetograms. ApJ 555, 448-461. doi:10.1086/321448

Howe, R., 2009. Solar Interior Rotation and its Variation. Living Reviews in Solar Physics 6, 1. doi:10.12942/lrsp-2009-1, arXiv:0902.2406

Howe, R., Christensen-Dalsgaard, J., Hill, F., Komm, R., Larson, T.P., Rempel, M., Schou, J., Thompson, M.J., 2013. The High-latitude Branch of the Solar Torsional Oscillation in the Rising Phase of Cycle 24. ApJ Lett. 767, L20. doi:10.1088/2041-8205/767/1/L20.

Howe, R., Komm, R., Hill, F., Ulrich, R., Haber, D.A., Hindman, B.W., Schou, J., Thompson, M.J., 2006. Large-Scale Zonal Flows Near the Solar Surface. Solar Phys. 235, 1-15. doi $10.1007 /$ s11207-006-0117-2.

Kaithakkal, A.J., Suematsu, Y., Kubo, M., Shiota, D., Tsuneta, S., 2013. The Association of Polar Faculae with Polar Magnetic Patches Examined with 
Hinode Observations. ApJ 776, 122. doi:10.1088/0004-637X/776/2/122, arXiv: 1311.0980

Leroy, J.L., Noens, J.C., 1983. Does the solar activity cycle extend over more than an 11-year period? A\&A 120, L1.

Martin, S.F., Harvey, K.H., 1979. Ephemeral active regions during solar minimum. Solar Phys. 64, 93-108. doi 10.1007/BF00151118.

McIntosh, S.W., Leamon, R.J., 2017. Deciphering Solar Magnetic Activity: Spotting Solar Cycle 25. ArXiv e-prints arXiv:1702.04414.

McIntosh, S.W., Wang, X., Leamon, R.J., Davey, A.R., Howe, R., Krista, L.D., Malanushenko, A.V., Markel, R.S., Cirtain, J.W., Gurman, J.B., Pesnell, W.D., Thompson, M.J., 2014. Deciphering Solar Magnetic Activity. I. On the Relationship between the Sunspot Cycle and the Evolution of Small Magnetic Features. ApJ 792, 12. doi:10.1088/0004-637X/792/1/12, arXiv:1403.3071

Minarovjech, M., Rušin, V., Saniga, M., 2011. The green corona database and the coronal index of solar activity. Contributions of the Astronomical Observatory Skalnate Pleso 41, 137-141.

Muñoz-Jaramillo, A., Sheeley, N.R., Zhang, J., DeLuca, E.E., 2012. Calibrating 100 Years of Polar Faculae Measurements: Implications for the Evolution of the Heliospheric Magnetic Field. ApJ 753, 146. doi:10.1088/0004-637X/ 753/2/146, arXiv:1303.0345.

Petrie, G.J.D., 2015. Solar Magnetism in the Polar Regions. Living Reviews in Solar Physics 12, 5. doi $10.1007 / \operatorname{lrsp}-2015-5$

Petrie, G.J.D., Petrovay, K., Schatten, K., 2014. Solar Polar Fields and the 22Year Activity Cycle: Observations and Models. Space Sci. Rev. 186, 325-357. doi: $10.1007 / \mathrm{s} 11214-014-0064-4$. 
Petrovay, K., 2010. Solar Cycle Prediction. Living Reviews in Solar Physics 7,

6. doi:10.12942/lrsp-2010-6, arXiv: 1012.5513.

Petrovay, K., Forgács-Dajka, E., 2002. The Role of Active Regions in the Generation of Torsional Oscillations. Solar Phys. 205, 39-52.

Priyal, M., Banerjee, D., Karak, B.B., Muñoz-Jaramillo, A., Ravindra, B., Choudhuri, A.R., Singh, J., 2014. Polar Network Index as a Magnetic Proxy for the Solar Cycle Studies. ApJ Lett. 793, L4. doi 10.1088/2041-8205/ 793/1/L4, arXiv: 1407.4944 .

Robbrecht, E., Wang, Y.M., Sheeley, Jr., N.R., Rich, N.B., 2010. On the "Extended" Solar Cycle in Coronal Emission. ApJ 716, 693-700. doi 10.1088/ 0004-637X/716/1/693.

Savcheva, A., Cirtain, J.W., DeLuca, E.E., Golub, L., 2009. Does a Polar Coronal Hole's Flux Emergence Follow a Hale-Like Law? ApJ Lett. 702, L32-L36. doi $10.1088 / 0004-637 X / 702 / 1 / L 32$.

Scherrer, P.H., Wilcox, J.M., Svalgaard, L., Duvall, Jr., T.L., Dittmer, P.H., Gustafson, E.K., 1977. The mean magnetic field of the sun - Observations at Stanford. Solar Phys. 54, 353-361. doi 10.1007/BF00159925.

Shiota, D., Tsuneta, S., Shimojo, M., Sako, N., Orozco Suárez, D., Ishikawa, R., 2012. Polar Field Reversal Observations with Hinode. ApJ 753, 157. doi:10.1088/0004-637X/753/2/157, arXiv:1205.2154,

SILSO WDC, 2017. The International Sunspot Number. International Sunspot Number Monthly Bulletin and online catalogue .

Spruit, H.C., 2003. Origin of the torsional oscillation pattern of solar rotation. Solar Phys. 213, 1-21. doi 10.1023/A:1023202605379, arXiv:astro-ph/0209146.

Sun, X., Hoeksema, J.T., Liu, Y., Zhao, J., 2015. On Polar Magnetic Field Reversal and Surface Flux Transport During Solar Cycle 24. ApJ 798, 114. doi:10.1088/0004-637X/798/2/114, arXiv:1410.8867. 
Tappin, S.J., Altrock, R.C., 2013. The Extended Solar Cycle Tracked High into the Corona. Solar Phys. 282, 249-261. doi 10.1007/s11207-012-0133-3. arXiv:1209.2969

Tlatov, A., Vasil'eva, V., Pevtsov, A., 2010. Distribution of magnetic bipoles on the sun over three solar cycles. Astrophys. J. 717, 357-362. doi 10.1088/ $0004-637 X / 717 / 1 / 357$.

Tlatov, A.G., Dormidontov, D.V., Kirpichev, R.V., Pashchenko, M.P., Shramko, A.D., Peshcherov, V.S., Grigoryev, V.M., Demidov, M.L., Svidskii, P.M., 2015. Study of some characteristics of large-scale solar magnetic fields during the global field polarity reversal according to observations at the telescopemagnetograph Kislovodsk Observatory. Geomagnetism and Aeronomy 55, 969-975. doi:10.1134/S0016793215070257.

Ulrich, R.K., Boyden, J.E., Webster, L., Padilla, S.P., Snodgrass, H.B., 1988. Solar rotation measurements at Mount Wilson. V - Reanalysis of 21 years of data. Solar Phys. 117, 291-328. doi 10.1007/BF00147250.

Waldmeier, M., 1935. Astr. Mitt. Zürich 14 (133), 105-130.

Wilson, P., Altrock, R., Harvey, K., Martin, S., Snodgrass, H., 1988. The extended solar activity cycle. Nature $333,748-+$. doi $10.1038 / 333748 \mathrm{a} 0$. 